\title{
Shape Effect of Silicon Nitride Subwavelength Structure on Reflectance for Silicon Solar Cells
}

\author{
Kartika Chandra Sahoo, Yiming Li, Member, IEEE, and Edward Yi Chang, Senior Member, IEEE
}

\begin{abstract}
In this paper, we, for the first time, examine the spectral reflectivity of hemisphere-, cone-, cylinder-, and parabolashaped silicon nitride $\left(\mathbf{S i}_{3} \mathbf{N}_{4}\right)$ subwavelength structures (SWSs). A multilayer rigorous coupled-wave approach is advanced to evaluate the reflection properties of $\mathrm{Si}_{3} \mathrm{~N}_{4}$ SWSs. We optimize the aforementioned four different shapes of SWSs in terms of effective reflectance over a range of wavelength. The results of our paper show that a lowest effective reflectivity could be achieved for the optimized cone-shaped SWS as compared to hemisphere-, parabola-, and cylinder-shaped structures with the same volume. The best shape SWS is then fabricated together with a silicon ( $\mathrm{Si})$ solar cell, and the efficiency of the solar cell is compared with that of a solar cell with single-layer antireflection coating (ARC). An increase of $1.09 \%$ in cell efficiency $(\eta)$ is observed for the Si solar cell with a cone-shaped $\mathrm{Si}_{3} \mathrm{~N}_{4} \mathrm{SWS}(\eta=12.86 \%)$ as compared with the cell with single-layer $\mathrm{Si}_{3} \mathrm{~N}_{4}$ ARCs $(\eta=11.77 \%)$.
\end{abstract}

Index Terms-Antireflection coating (ARC), efficiency, morphological effect, multilayer, reflectance, rigorous coupled-wave approach, shape effect, silicon nitride, subwavelength structure (SWS).

\section{INTRODUCTION}

D UE TO THE high refractive index contrast between silicon and air, the surface of the polished silicon $(\mathrm{Si})$ wafer reflects 36\% incident light. Lowering the surface reflectivity of Si by texturization is one of the most important processes for improving the conversion efficiency of Si solar cells. By developing the surface texture on a Si substrate, the following three effects can be observed: 1) reduction in surface reflection; 2 ) increase in light absorption due to an increase in optical path length by diffraction; and 3) enhancement of internal reflection that reduces the amount of escaping light. The first effect is essential for increasing the input energy to solar cells, irrespective of the cell thickness. So far, various surface texturing

Manuscript received March 31, 2010; revised June 9, 2010; accepted June 23, 2010. Date of publication August 3, 2010; date of current version September 22, 2010. This work was supported in part by the National Science Council of Taiwan under Contracts NSC-97-2221-E-009-154-MY2 and NSC98-2120-M-009-010, by Chimei Innolux Corporation, Chunan Science Park, Miao-li, Taiwan, under a 2009-2011 grant, and by Motech Industries Inc. (Motech), Tainan, Taiwan, under a 2008-2009 grant. The review of this paper was arranged by Editor A. Aberle.

K. C. Sahoo is with the Department of Electrical Engineering, National Chiao Tung University, Hsinchu 300, Taiwan.

$\mathrm{Y}$. Li is with the Department of Electrical Engineering, National Chiao Tung University, Hsinchu 300, Taiwan, and also with the National Nano Device Laboratories, Hsinchu 300, Taiwan (e-mail: ymli@ faculty.nctu.edu.tw).

E. Y. Chang is with the Department of Materials Science and Engineering, National Chiao Tung University, Hsinchu 300, Taiwan.

Color versions of one or more of the figures in this paper are available online at http://ieeexplore.ieee.org.

Digital Object Identifier 10.1109/TED.2010.2056150 techniques have been tried on crystalline silicon, including laser structuring [1], mechanical diamond saw cutting [2], and maskless reactive-ion etching (RIE) processing [3]. If we observe the maskless RIE processing, several self-assembly masks, such as anodic porous aluminum [4], [5] spin-coated spheres [6]-[9], and evaporated Ag islands [10], followed by RIE, have been demonstrated. Among these methods, RIE provides high rates of isotropic etching. However, this may form dislocations and defects in semiconductor layer [11]. These defects and dislocations are responsible for increasing the minority carrier recombination in a solar cell [12]. Thus, the short-circuit current for the solar cell is decreased, which, in turn, decreases the solar cell efficiency. To reduce the probability of defect creation in the semiconductor layer, the subwavelength structure (SWS) on antireflection coating (ARC) instead of semiconductor surface has been studied recently [13]-[16] by our group. In our recent papers, we have advanced a rigorous coupled-wave analysis (RCWA) method to study the reflectance of $\mathrm{Si}_{3} \mathrm{~N}_{4}$ SWS for a wavelength ranging from 400 to $1000 \mathrm{~nm}$ using an effective refractive index calculated for a pyramidal shape using effective medium theory (EMT) [13]. To introduce the effective refractive index gradient required for antireflection applications, SWSs with tapered profiles and high aspect ratios are highly desirable [17]. We found that $\mathrm{Si}_{3} \mathrm{~N}_{4}$ SWSs with coneand pillarlike structures can successfully be fabricated using a self-assembled nickel nanocluster, followed by an inductively coupled plasma etching method, and the reflectance spectra of these subwavelength structures depend on their shapes [14]. Therefore, it is worth studying the shape effect of $\mathrm{Si}_{3} \mathrm{~N}_{4}$ SWS on reflectance to enhance the solar cell efficiency for us.

In this paper, we study the reflectance of SWSs with four different shapes (i.e., hemisphere, cone, cylinder, and parabola) on silicon nitride $\left(\mathrm{Si}_{3} \mathrm{~N}_{4}\right)$ for solar cell application. The main motivation behind this is to choose optimal shapes of $\mathrm{Si}_{3} \mathrm{~N}_{4}$ SWSs with the lowest reflectance that can be used in Si solar cells. Solar cells with the explored silicon nitride SWS on them are thus fabricated for the first time, and the results are presented and discussed. A multilayer RCWA [18]-[20] is advanced to investigate the reflection properties of $\mathrm{Si}_{3} \mathrm{~N}_{4}$ SWSs. We first optimize $\mathrm{Si}_{3} \mathrm{~N}_{4}$ SWSs with hemisphere-, cone-, cylinder-, and parabola-shaped structures for the best effective reflectance properties. Using the optimized morphologies, we further compare the results of reflectance among these shapes of SWSs by considering the wavelength range from 400 to $1000 \mathrm{~nm}$. Consequently, Si solar cells with an optimized shape of $\mathrm{Si}_{3} \mathrm{~N}_{4}$ SWS are fabricated using a conventional solar cell process incorporated with the $\mathrm{Si}_{3} \mathrm{~N}_{4}$ SWS fabrication method reported earlier [14]. 


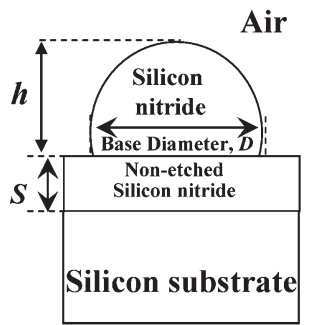

(a)

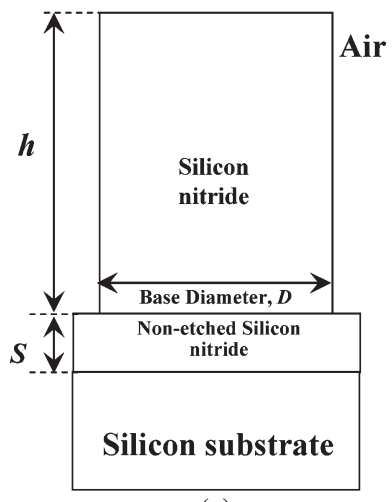

(c)

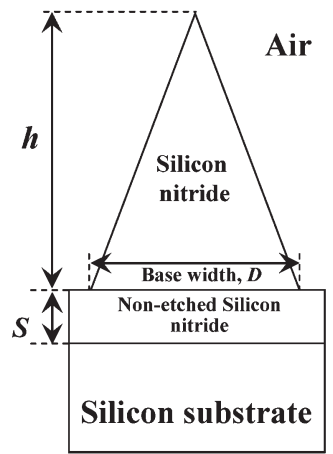

(b)

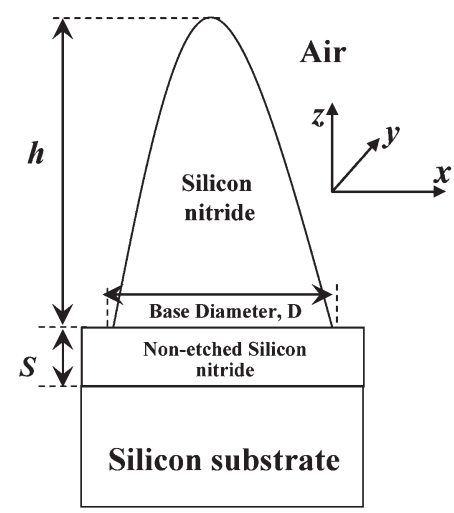

(d)
Fig. 1. Schematic of the SWS studied in this paper, where $h$ is the height and $s$ is the nonetched part of SWS with (a) hemisphere-, (b) cone-, (c) cylinder-, and (d) parabola-shaped structures.

This paper is organized as follows. In Section II, we brief the procedure of RCWA for the simulated SWS structures, including the adopted material parameters, and discuss the simulation results. In Section III, we show the procedure for fabrication of Si solar cells, followed by the fabrication results. Finally, we draw conclusions and suggest future work.

\section{Calculation Method and Results}

We study the SWS structure with four different shapes, as shown in Fig. 1, for the reflectance property with respect to wavelength. We choose these shapes because all shapes are of cylindrical symmetry with the same base radius. The height of $\mathrm{Si}_{3} \mathrm{~N}_{4} \mathrm{SWS}$ is $h$, and the thickness of the nonetched $\mathrm{Si}_{3} \mathrm{~N}_{4}$ layer is $s$. Both of these parameters are the key designing parameters for reflectance optimization. We use RCWA [13] to study the diffractive structure and its reflectance property, where EMT [21]-[23] is adopted to calculate the effective refractive index for each partitioned uniform homogeneous layer. We first divide the SWS structure into several horizontal layers with equal thickness, and for each discrete position $z_{l}$ along the $z$-direction, EMT implies that the effective refractive index $n\left(z_{l}\right)$ of each layer is approximated by (1), shown at the bottom of the page, where $f\left(z_{l}\right)$ is the fraction of $\mathrm{Si}_{3} \mathrm{~N}_{4}$ contained in each layer given by $f\left(z_{l}\right)=\left(\pi r_{l}^{2} / \sqrt{3} D^{2}\right)$, with $r_{l}$ being calculated according to the shape of SWS [24]. The $r_{l}$ for hemisphere-, parabola-, cone-, and cylinder-shaped SWSs are given by the following equations, respectively:

$$
\begin{aligned}
& r_{l}=\sqrt{r^{2}-\left(z_{l}+r-h\right)^{2}} \\
& r_{l}=r \sqrt{\left(1-\frac{z_{l}}{h}\right)} \\
& r_{l}=r\left(1-\frac{z_{l}}{h}\right) \\
& r_{l}=r .
\end{aligned}
$$

Notably, $n_{\mathrm{SiN}}=n+i k$ is the complex refractive index of $\mathrm{Si}_{3} \mathrm{~N}_{4}$, where $i=\sqrt{-1} ; n$ and $k$ are optical constants; and $n_{\text {air }}=1$ is the refractive index of air. Only the real part of the refractive index of $\mathrm{Si}_{3} \mathrm{~N}_{4}$ is considered in our simulation because it is a weakly absorbing material above $400 \mathrm{~nm}$ [25]. With the calculated effective refractive index $n\left(z_{l}\right)$ for each layer, we can calculate the reflectance of the entire structure including a layer for the nonetched $\mathrm{Si}_{3} \mathrm{~N}_{4}$ with respect to the different wavelengths [13]. Here, the incident angle $\theta$ of sunlight is assumed to be normal to the plane (i.e., $\theta=0^{\circ}$ ), and only TE polarization is considered here for the calculation of reflectance [26].

Instead of considering the reflectance for a certain wavelength, an effective reflectance is computed for the structures over a range of the wavelength of incident sunlight. By taking $s$ and $h$ as varying factors, we calculate the effective reflectance $R_{\text {eff }}$ [27] for the wavelength $\lambda$ varying from $\lambda_{l}=400 \mathrm{~nm}$ to $\lambda_{u}=1000 \mathrm{~nm}$ and compare it with $\mathrm{Si}_{3} \mathrm{~N}_{4}$ SWS. $R_{\text {eff }}$ is evaluated by

$$
R_{\mathrm{eff}}=\frac{\int_{\lambda_{l}}^{\lambda_{u}} \frac{R(\lambda) S I(\lambda)}{E(\lambda)} d \lambda}{\int_{\lambda_{l}}^{\lambda_{u}} \frac{S I(\lambda)}{E(\lambda)} d \lambda}
$$

where $S I(\lambda)$ is spectral irradiance given by ATMG173 AM1.5G reference [28], $E(\lambda)$ is the photon energy, and $R(\lambda)$ is the calculated reflection. The " $s$ " and " $h$ " factors are varied for the studied structures to achieve the lowest effective reflectance, and the optimization results are shown in Fig. 2. The reflectance spectra of the optimized structures are compared in Fig. 3. The lowest effective reflectance of $1.93 \%$ is observed for the optimized parabola-shaped SWS as compared to the results of hemisphere-, cone-, and cylinder-shaped structures.

Comparison of the reflectance spectra of the four shapes, as shown in Fig. 3, may not be correct as the volumes of the different shapes are different. For this reason, we keep

$$
n\left(z_{l}\right)=\sqrt{\frac{\left[1-f\left(z_{l}\right)+f\left(z_{l}\right) n_{\mathrm{SiN}}^{2}\right]\left[f\left(z_{l}\right)+\left(1-f\left(z_{l}\right)\right) n_{\mathrm{SiN}}^{2}\right]+n_{\mathrm{SiN}}^{2}}{2\left[f\left(z_{l}\right)+\left(1-f\left(z_{l}\right)\right) n_{\mathrm{SiN}}^{2}\right]}}
$$




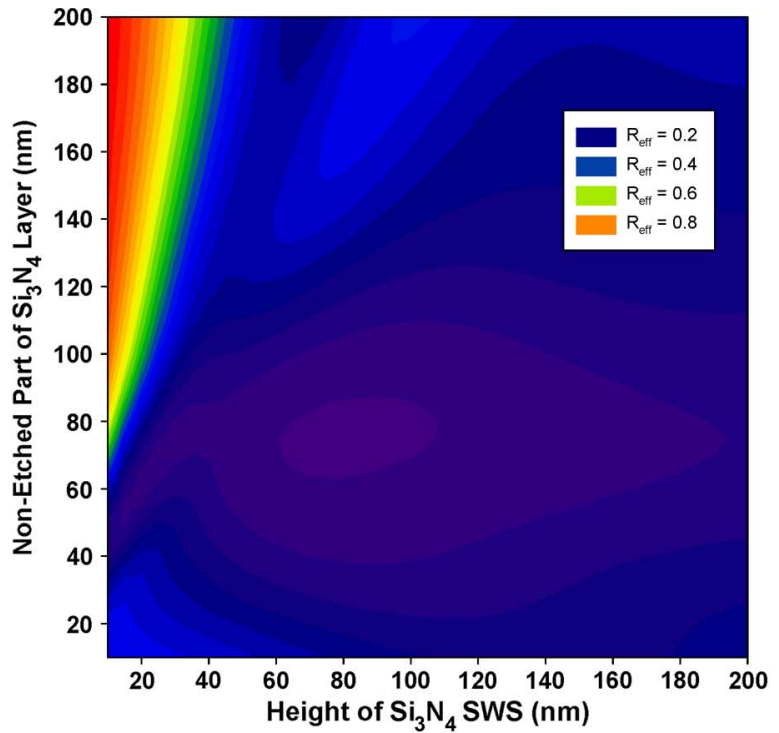

(a)

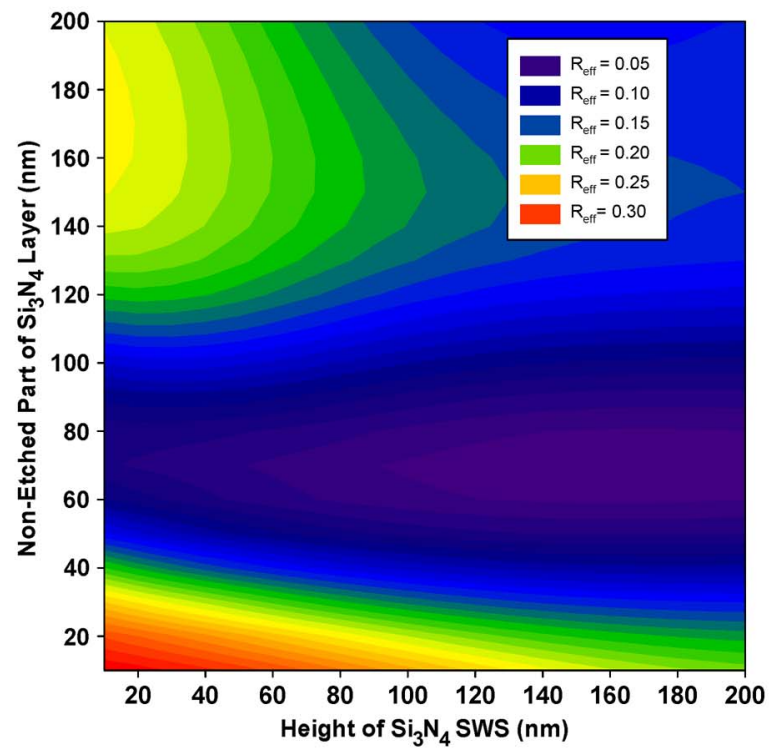

(c)

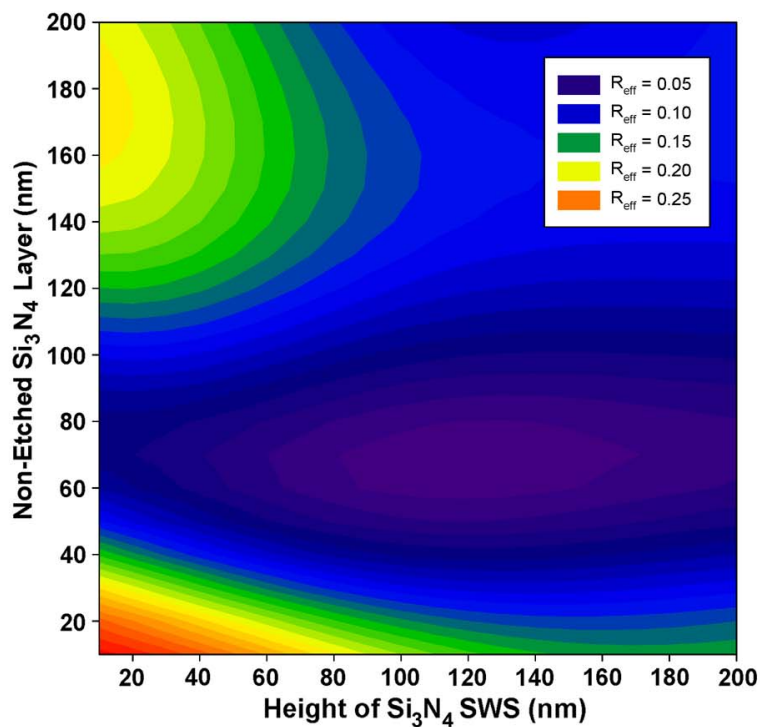

(b)

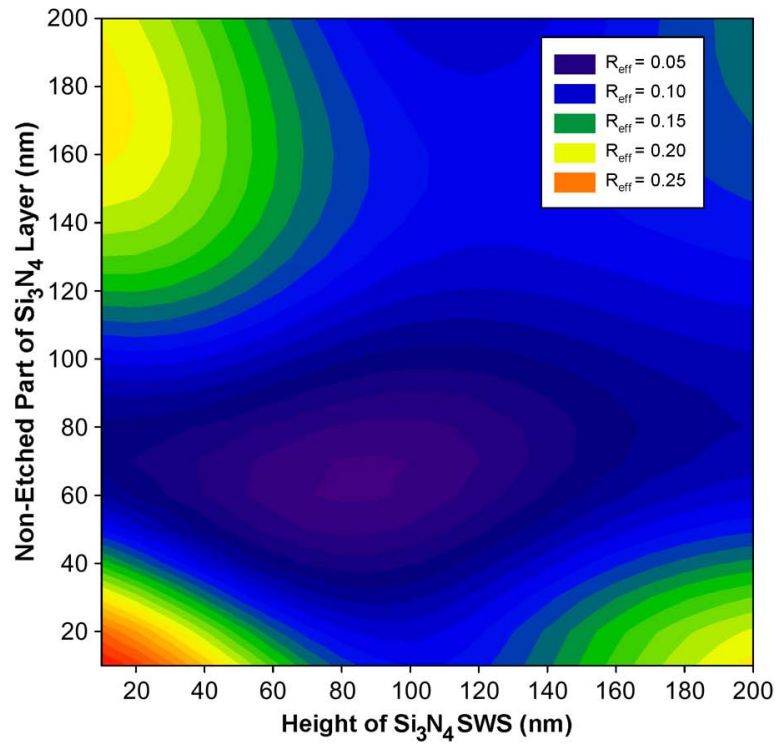

(d)

Fig. 2. Contour plot of the effective reflectance for the wavelength varying from 400 to $1000 \mathrm{~nm}$; the plot is as a function of $h$ and $s$ for $\mathrm{Si}_{3} \mathrm{~N}_{4} \mathrm{SWS}_{\mathrm{S}}$ for (a) hemisphere-, (b) parabola-, (c) cone-, and (d) cylinder-shaped structures.

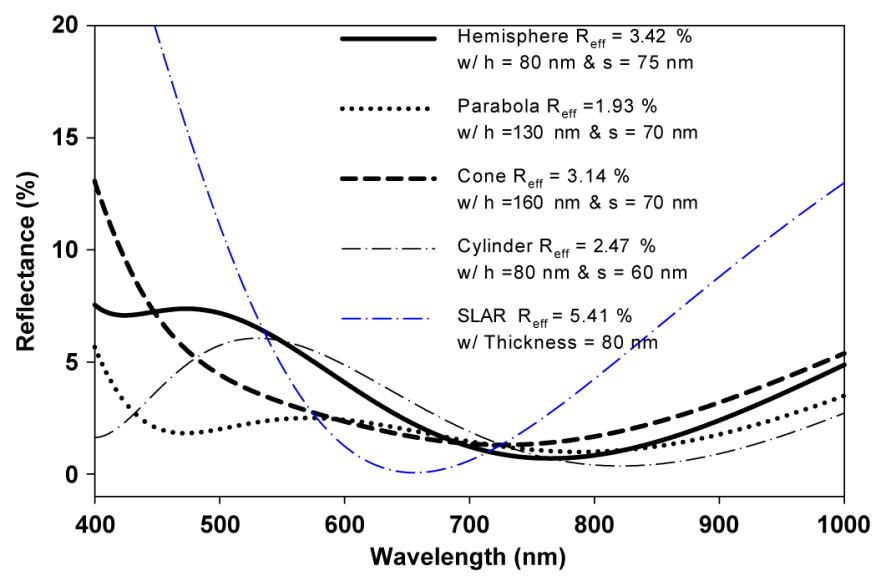

Fig. 3. Comparison of the reflectance spectra among the optimized $\mathrm{Si}_{3} \mathrm{~N}_{4}$ structures for the wavelength varying from 400 to $1000 \mathrm{~nm}$.

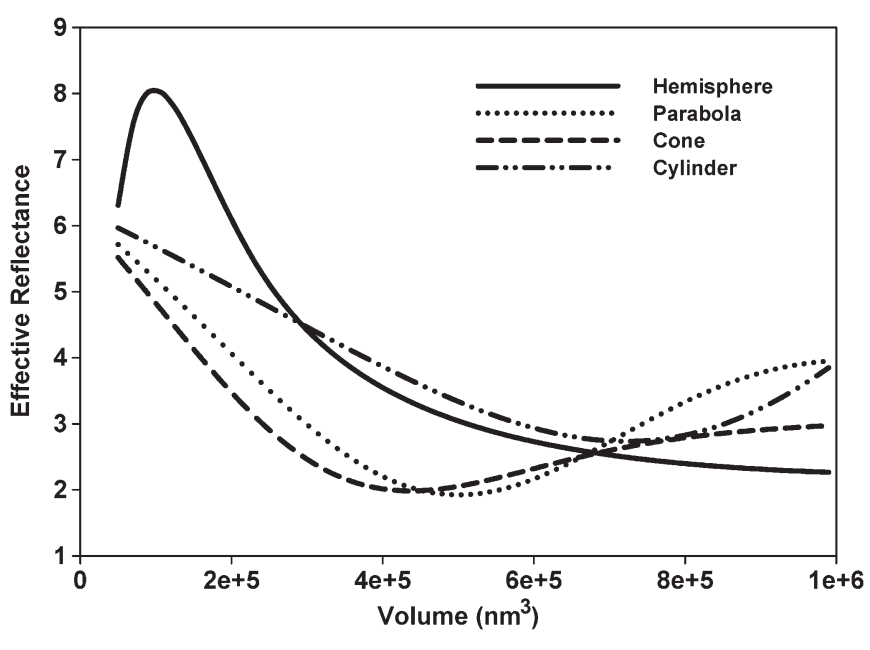

Fig. 4. Plot of the effective reflectance with volume for four different $\mathrm{Si}_{3} \mathrm{~N}_{4}$ structures for the wavelength varying from 400 to $1000 \mathrm{~nm}$. 

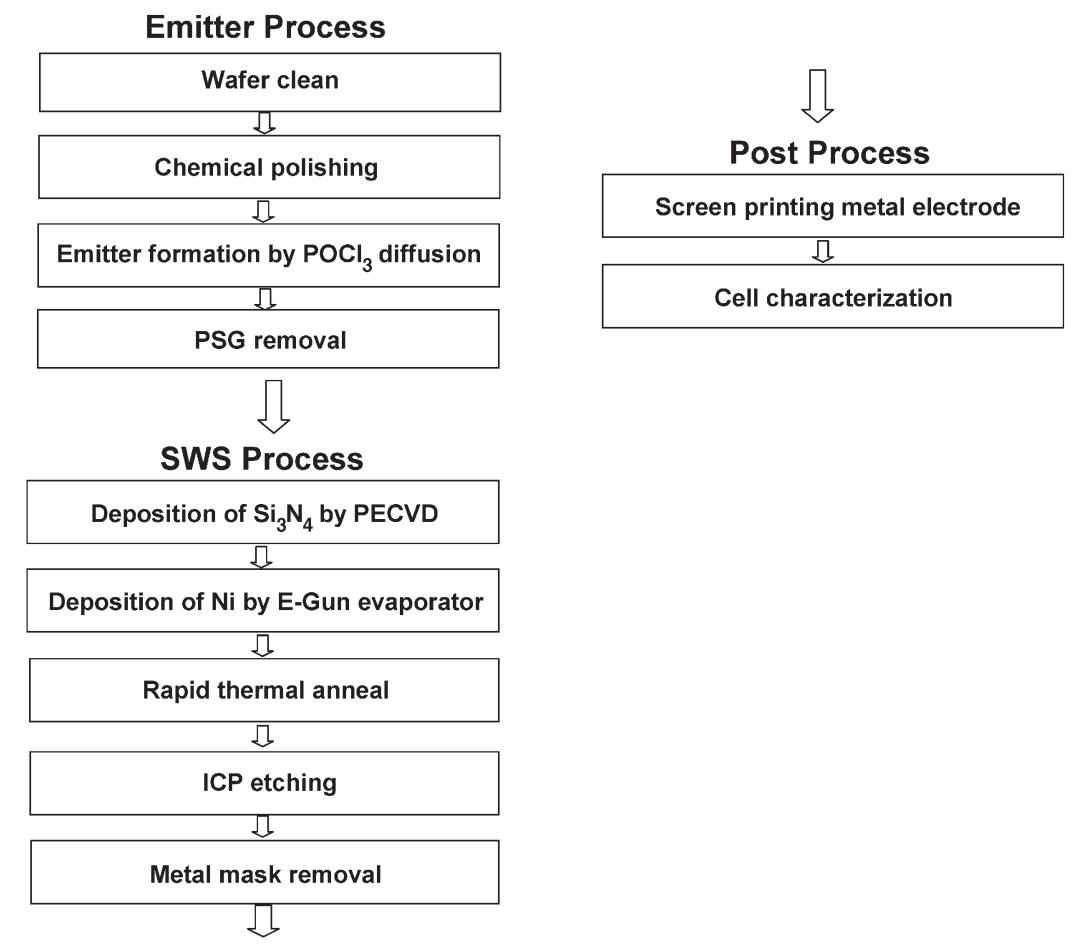

Fig. 5. $\mathrm{Si}_{3} \mathrm{~N}_{4}$ SWS solar cell process flow. (a) Emitter process. (b) SWS process. (c) Postprocess.

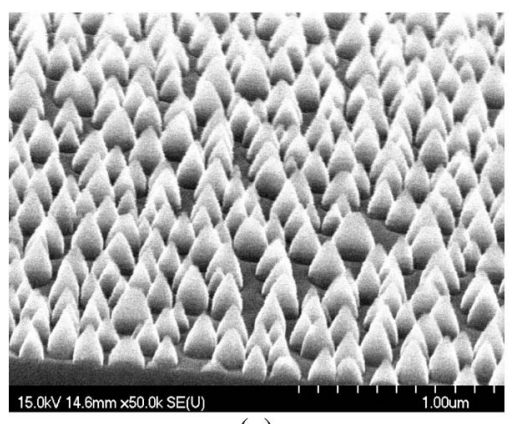

(a)

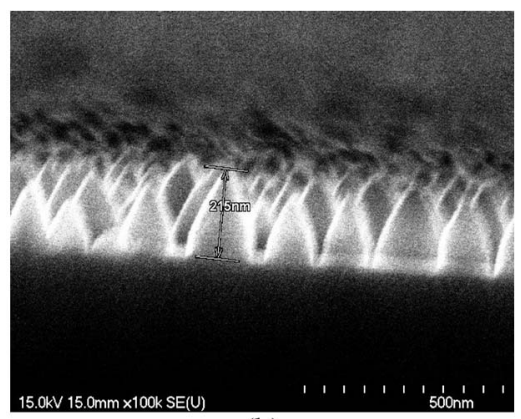

(b)

Fig. 6. Scanning electron microscope image of $\mathrm{Si}_{3} \mathrm{~N}_{4}$ nanocone structures. (a) Top view. (b) Cross-sectional view.

the volume constant for all the shapes and vary the height of SWS to see the effect on reflectance. Note that when we change the SWS height, the thickness of the nonetched $\mathrm{Si}_{3} \mathrm{~N}_{4}$ SWS is kept constant at $70 \mathrm{~nm}$. The effective reflectances of the hemisphere-, cone-, cylinder-, and parabola-shaped $\mathrm{Si}_{3} \mathrm{~N}_{4}$ SWSs with different volumes are shown in Fig. 4. For the volume varying from $5 \times 10^{4}$ to $1 \times 10^{6} \mathrm{~nm}^{3}$, we find that the cone-shaped SWS has the lowest effective reflectance and that the cylinder-shaped SWS has the highest effective reflectance as compared to other shapes.

\section{SAMPLE FABRICATION AND CHARACTERIZATION}

Based on our observation in our simulation, we fabricate a $\mathrm{Si}$ solar cell with $\mathrm{Si}_{3} \mathrm{~N}_{4}$ nanocone structures for the first time. The flowchart of the fabrication process for a solar cell with silicon nitride SWS is shown in Fig. 5. First, a p-type silicon wafer is cleaned with $\mathrm{H}_{2} \mathrm{SO}_{4} / \mathrm{H}_{2} \mathrm{O}_{2}$ and then followed by chemical polishing to remove the surface damage. Then, the emitter is formed by phosphorous diffusion by supplying phosphorous trichloride oxide $\left(\mathrm{POCl}_{3}\right)$ to the silicon wafer. In this process, p-type silicon wafer is loaded into a quartz boat, which was slowly moved into the middle of a fused quartz tube in a resistance-heated horizontal furnace. The furnace temperature for the diffusion was held at about $900{ }^{\circ} \mathrm{C}$. Nitrogen is used as a carrier gas. During the diffusion process, the following reactions take place to form a phosphor silicate glass (PSG):

$$
\begin{aligned}
4 \mathrm{POCl}_{3}+3 \mathrm{O}_{2} & \rightarrow 2 \mathrm{P}_{2} \mathrm{O}_{5}+6 \mathrm{Cl}_{2} \\
2 \mathrm{P}_{2} \mathrm{O}_{5}+5 \mathrm{Si} & \rightarrow 4 \mathrm{P}+5 \mathrm{SiO}_{2} .
\end{aligned}
$$

When a PSG is deposited on the silicon substrate, phosphorus starts to diffuse into the bulk, and the emitter is formed. Then, the PSG is removed by dipping the wafer in a $10 \%$ buffered HF solution for about $1 \mathrm{~min}$. Then, the silicon nitride cone-shaped structures are formed on the front surface of the solar cell using the fabrication process reported in [15], which is briefly described in Fig. 5. The scanning electron microscope image of the fabricated nanocone structure is shown in Fig. 6. After 

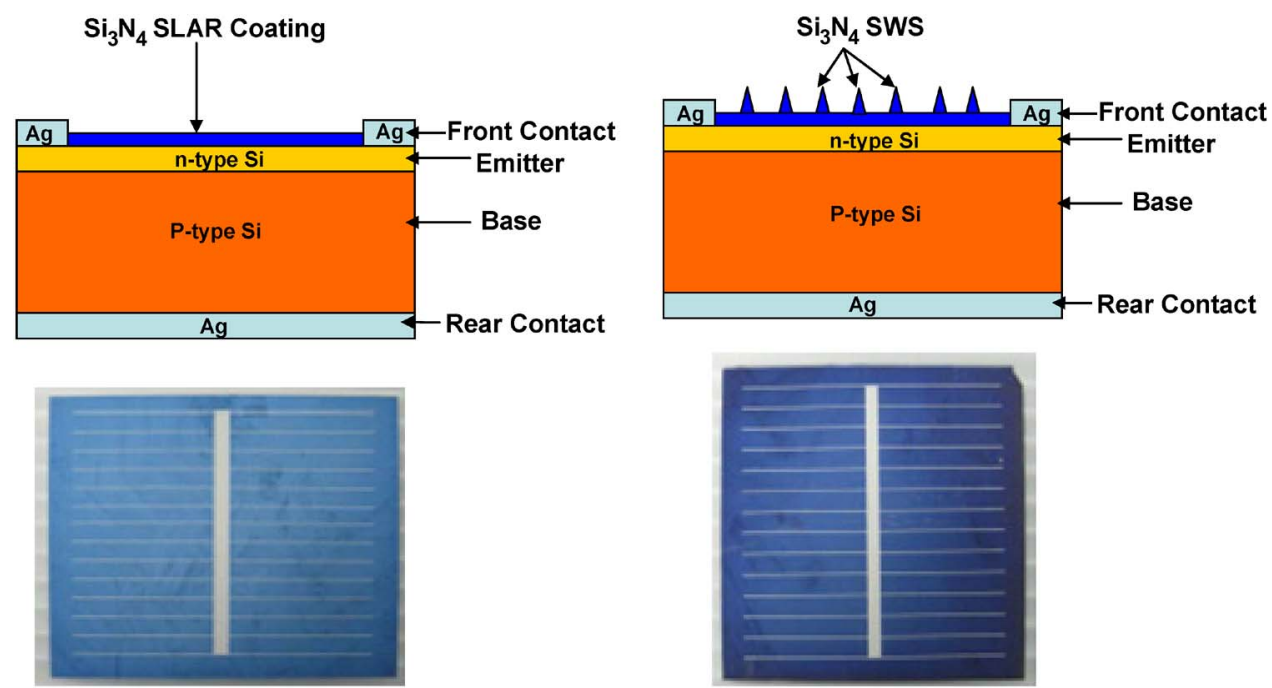

(a)

(b)

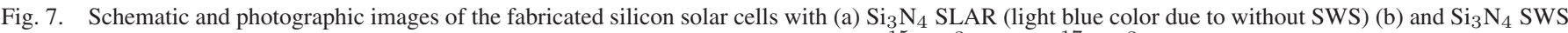
(dark blue due to SWS). For both samples, the doping level of $0.5-\mu \mathrm{m}$-thick n-type $\mathrm{Si}$ is $10^{15} / \mathrm{cm}^{3}$ and is $10^{17} / \mathrm{cm}^{3}$ for p-type $\mathrm{Si}$, where the substrate resistivity is $1 \Omega \cdot \mathrm{cm}$.

TABLE I

Measured Solar Cell $I-V$ Data for Silicon Nitride SLAR and Silicon Nitride SWSs. The Difference of Electrical Characteristics

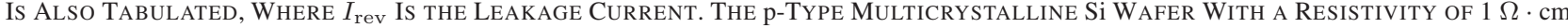

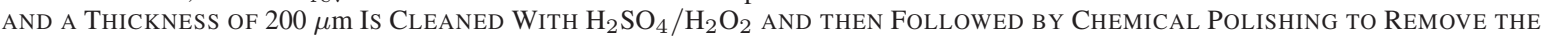
Surface Damage. When a PSG Is Deposited on the Si Substrate, Phosphorus Starts to Diffuse Into the Bulk, AND the Emitter Is Formed With a Junction Depth of $0.6 \mu \mathrm{m}$. The Area Is $1 \mathrm{~cm}^{2}$

\begin{tabular}{|c|c|c|c|c|c|}
\hline & $\mathrm{V}_{\mathrm{OC}}(\mathrm{V})$ & $\mathrm{J}_{\mathrm{SC}}\left(\mathrm{mA} / \mathrm{cm}^{2}\right)$ & $\mathrm{FF}(\%)$ & Efficiency (\%) & $\mathrm{I}_{\text {rev }}(\mathrm{A})$ \\
\hline $\mathrm{Si}_{3} \mathrm{~N}_{4} \mathrm{SLAR}$ & 0.596 & 30.8 & 64.24 & 11.77 & 0.22 \\
\hline $\mathrm{Si}_{3} \mathrm{~N}_{4} \mathrm{SWS}$ & 0.595 & 33.7 & 64.2 & 12.86 & 0.63 \\
\hline $\begin{array}{c}\text { Difference between } \mathrm{Si}_{3} \mathrm{~N}_{4} \\
\mathrm{SLAR} \text { and } \mathrm{Si}_{3} \mathrm{~N}_{4} \mathrm{SWS}\end{array}$ & -0.001 & +2.9 & -0.04 & $+\mathbf{1 . 0 9}$ & +0.41 \\
\hline
\end{tabular}

the SWS process, the postprocessing of the cell is done, which includes electrode formation by the standard screen-printing method and the cell characterization to get the open-circuit voltage $\left(V_{\mathrm{OC}}\right)$, short-circuit current density $\left(J_{\mathrm{SC}}\right)$, efficiency $(\eta)$, and fill factor (FF) under AM1.5G conditions. The Ag and silver-aluminum $(\mathrm{Ag} / \mathrm{Al})$ contacts on the front $(\mathrm{F})$ and back $(\mathrm{B})$ surfaces of the cells, respectively, are made by screen printing, followed by sintering at $650{ }^{\circ} \mathrm{C}-750{ }^{\circ} \mathrm{C}$ for a few minutes. For the sintering process, the screen-printed wafers are placed vertically in a fused silica boat in the FFBBFFBB configuration. This boat is then placed in the hot zone of the sintering furnace for a few minutes in order to carry out the sintering in zero air or equivalent $\mathrm{N}_{2} / \mathrm{O}_{2}$ ambient.

The schematic and the photographic images of the fabricated solar cell with single-layer antireflection (SLAR) and coneshaped $\mathrm{Si}_{3} \mathrm{~N}_{4}$ SWSs are shown in Fig. 7(a) and (b), respectively. The color of the solar cell with $\mathrm{Si}_{3} \mathrm{~N}_{4}$ SWS is dark blue as compared with the solar cell without SWS, which is similar to the case of single-crystalline silicon without and with etching reported in [29]. The measured data of current-voltage $(I-V)$ of the fabricated sample are tabulated in Table I, where the fabricated Si solar cell with silicon nitride SWS and the Si solar cell with SLAR coating are compared in Table I. It is observed that the $V_{\mathrm{OC}}$ value of the SWS solar cell is decreased by $0.001 \mathrm{~V}$ as compared to that of the SLAR solar cell. Also, the FF of the
SWS solar cell is decreased by $0.04 \%$ as compared to that of the SLAR solar cell. However, the $J_{\mathrm{SC}}$ and efficiency of SWS have been improved by $2.9 \mathrm{~mA} / \mathrm{cm}^{2}$ and $1.09 \%$ as compared to that of the SLAR solar cell. The decrease of FF suggests that there must be some insufficient electrical contact, which is also confirmed by the high reverse current of the SWS solar cell compared to that of the SLAR solar cell. Thus, there is a need to improve the fabrication process, specifically for the postprocess steps of Fig. 5. The increase in efficiency by $1.09 \%$ for the SWS solar cell has been achieved compared to that of the silicon solar cell with SLAR. We expect that the efficiency can be further improved more if the electrode formation step could be improved for the Si solar cell with silicon nitride SWS.

\section{CONCLUSION}

In this paper, we have compared four different designed silicon nitride SWSs. Using the results of rigorous coupledwave analysis simulation for the hemisphere-, cone-, cylinder-, and parabola-shaped silicon nitride SWSs, the ratio of silicon nitride SWS height to the nontextured part of silicon nitride has been optimized. The reflectance results for the optimized SWSs have been compared in terms of effective reflectivity. The cone-shaped silicon nitride SWS has been observed to be best suited for a solar cell as compared to the results 
of hemisphere-, cylinder-, and parabola-shaped silicon nitride SWSs. A silicon solar cell with a cone-shaped silicon nitride SWS has been fabricated successfully, and the results show the clear increase of $1.09 \%$ in efficiency as compared with a solar cell with silicon nitride SLAR. In addition, there is a need to improve the electrical contact process for a silicon nitride SWS to further increase the efficiency, as seen from the measurement. We expect that the efficiency can be improved more if the postprocess steps of the silicon solar cell with a silicon nitride SWS can be improved significantly.

\section{ACKNOWLEDGMENT}

K. C. Sahoo would like to thank for the sample fabrication and characterization from MOTECH.

\section{REFERENCES}

[1] S. Narayanan, "High efficiency polycrystalline silicon solar cell," Ph.D. dissertation, Univ. New South Wales, Sydney, Australia, May, 1989.

[2] P. Path, G. Wileke, E. Bucher, J. Szlufcic, R. M. Murti, K. DeClercq, J. Nijs, and R. Mertens, "Mechanical wafer engineering for high efficiency solar cells: An investigation of the induced surface damage," in Proc. 24th IEEE Photovoltaic Spec. Conf., 1994, pp. 1347-1350.

[3] S. H. Zaidi, D. S. Ruby, and J. M. Gee, "Characterization of random reactive ion etched-textured silicon solar cells," IEEE Trans. Electron Devices, vol. 48, no. 6, pp. 1200-1206, Jun. 2001.

[4] Y. Kanamori, K. Hane, H. Sai, and H. Yugami, "100 nm period silicon antireflection structures fabricated using a porous alumina membrane mask," Appl. Phys. Lett., vol. 78, no. 2, pp. 142-144, Jan. 2001.

[5] H. Sai, H. Fujii, K. Arafune, Y. Ohshita, M. Yamaguchi, Y. Kanamori, and H. Yugami, "Antireflective subwavelength structures on crystalline Si fabricated using directly formed anodic porous alumina masks," Appl. Phys. Lett., vol. 88, no. 20, pp. 201 116-1-201 116-3, May 2006.

[6] C. H. Sun, W. L. Min, N. C. Linn, P. Jiang, and B. Jiang, "Templated fabrication of large area subwavelength antireflection gratings on silicon," Appl. Phys. Lett., vol. 91, no. 23, pp. 231 105-1-231 105-3, Dec. 2007.

[7] C. H. Sun, P. Jiang, and B. Jiang, "Broadband moth-eye antireflection coatings on silicon," Appl. Phys. Lett., vol. 92, no. 6, pp. 061 112-1061 112-3, Feb. 2008.

[8] C. H. Sun, A. Gonzalez, N. C. Linn, P. Jiang, and B. Jiang, "Bioinspired broadband antireflection coatings on GaSb," Appl. Phys. Lett., vol. 92, no. 14 , pp. 141 109-1-141 109-3, Apr. 2008.

[9] W. L. Min, A. P. Betancourt, P. Jiang, and B. Jiang, "Templated biomimetic multifunctional coatings," Appl. Phys. Lett., vol. 92, no. 5, pp. 051 107-1-051 107-3, Feb. 2008.

[10] S. Wang, X. Z. Yu, and H. T. Fan, "Simple lithographic approach for subwavelength structure antireflection," Appl. Phys. Lett., vol. 91, no. 6, pp. 061 105-1-061 105-3, Aug. 2007.

[11] G. Kumaravelu, M. M. Alkaisi, A. Bittar, D. Macdonald, and J. Zhao, "Damage studies in dry etched textured silicon surfaces," Curr. Appl. Phys., vol. 4, no. 2-4, pp. 108-110, Apr. 2004.

[12] B. L. Sopori, Y. Zhang, and R. Reedy, "H diffusion for impurity and defect passivation: A physical model for solar cell processing," in Proc. 29th IEEE PV Spec. Conf., May 20-24, 2002, pp. 1-5.

[13] K. C. Sahoo, Y. Li, and E. Y. Chang, "Numerical calculation of reflectance of sub-wavelength structures on silicon nitride for solar cell application," Comput. Phys. Commun., vol. 180, no. 10, pp. 1721-1729, Oct. 2009.

[14] K. C. Sahoo, M. K. Lin, E. Y. Chang, Y. Y. Lu, C. C. Chen, J. H. Huang, and C. W. Chang, "Fabrication of antireflective sub-wavelength structures on silicon nitride using nano cluster mask for solar cell application," Nanoscale Res. Lett., vol. 4, no. 7, pp. 680-683, Jul. 2009.

[15] K. C. Sahoo, M. K. Lin, E. Y. Chang, T. B. Tinh, Y. Li, and J. H. Huang, "Silicon nitride nanopillars and nanocones formed by nickel nanoclusters and inductively coupled plasma etching for solar cell application," Jpn. J. Appl. Phys., vol. 48, no. 12, pp. 126 508-1-126 508-4, Dec. 2009.

[16] K. C. Sahoo, Y. Li, M.-K. Lin, E. Y. Chang, and J.-H. Huang, "Design and fabrication of sub-wavelength structure on silicon nitride for solar cell application," in Proc. IEEE 9th Nanotechnol. Conf., Geneoa, Italy, 2009, pp. $127-130$.

[17] S. J. Wilson and M. C. Hutley, "The optical properties of 'moth eye' antireflection surfaces," Opt. Acta, vol. 29, no. 7, pp. 993-1009, Jul. 1982.
[18] M. G. Moharam and T. K. Gaylord, "Rigorous coupled-wave analysis of planar-grating diffraction," J. Opt. Soc. Amer, vol. 71, no. 7, pp. 811-818, Jul. 1981.

[19] M. G. Moharam and T. K. Gaylord, "Rigorous coupled-wave analysis of metallic surface-relief gratings," J. Opt. Soc. Amer. A, Opt. Image Sci., vol. 3, no. 11, pp. 1780-1787, Nov. 1986.

[20] M. G. Moharam and D. A. Pommet, "Formulation for stable and efficient implementation of the rigorous coupled-wave analysis of binary gratings," J. Opt. Soc. Amer. A, Opt. Image Sci., vol. 12, no. 5, pp. 1077-1086, May 1995.

[21] L. Lalanne and M. Hutley, Artificial Media Optical PropertiesSubwavelength Scale. New York: Marcel Dekker, 2003.

[22] P. Yeh, Optical Waves in Layered Media. New York: Wiley, 1991.

[23] D. A. G. Bruggeman, "Dielectric constant and conductivity of mixtures of isotropic materials," Ann. Phys., vol. 24, pp. 636-679, 1935.

[24] M. E. Motamedi, W. H. Southwell, and W. J. Gunning, "Antireflection surfaces in silicon using binary optics technology," Appl. Opt., vol. 31, no. 22, pp. 4371-4376, 1992.

[25] J. Zhao and M. A. Green, "Optimized anti-reflection coatings for highefficiency silicon solar cells," IEEE Trans. Electron Devices, vol. 38, no. 8, pp. 1925-1934, Aug. 1991.

[26] K. L. Chopra and S. R. Das, Thin Film Solar Cells. New York: Plenum, 1983.

[27] D. N. Wright, E. S. Marstein, and A. Holt, "Acidic texturing of multicrystalline silicon wafers," in Proc. 31st IEEE Photovoltiac Spec. Conf., Orlando, FL, 2005, pp. 1237-1240.

[28] [Online]. Available: http://rredc.nrel.gov/solar/spectra/am1.5/ASTMG173 html

[29] K. Nishioka, S. Horita, and K. Ohdaria, "Anti-reflection sub-wavelength structure of silicon surface formed by wet process using catalysis of single nano-sized gold particle," Sol. Energy Mater. Sol. Cells, vol. 92, no. 8, pp. 919-922, Aug. 2008.

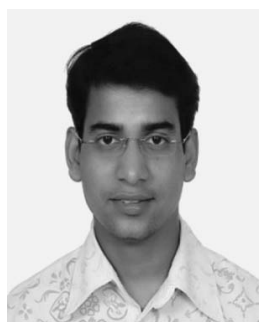

Kartika Chandra Sahoo received the B.S. degree in physics and the M.S. degree in electronics from Berhampur University, Berhampur, India, in 1998 and 2000, respectively, and the Ph.D. degree from the Department of Materials Science and Engineering, National Chiao Tung University (NCTU), Hsinchu, Taiwan, in 2009.

He is currently a Postdoctoral Fellow in the Parallel and Scientific Computing Laboratory, Department of Electrical Engineering, NCTU. His research interests include solar cell devices, advanced semiconductor devices, nanotechnology, semiconductor device simulation: model and programming, and TCAD simulation of CMOS and HEMT devices. 


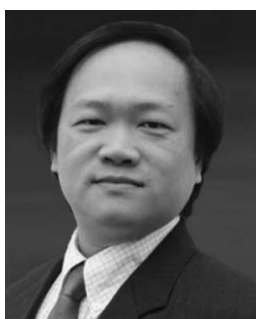

Yiming Li (M'02) received the B.S. degree in applied mathematics and electronics engineering, the M.S. degree in applied mathematics, and the Ph.D. degree in electronics from National Chiao Tung University (NCTU), Hsinchu, Taiwan, in 1996, 1998, and 2001, respectively.

In 2001, he joined the National Nano Device Laboratories (NDL), Hsinchu, as an Associate Researcher and the Microelectronics and Information Systems Research Center (MISRC), NCTU, as a Research Assistant Professor, where he has been engaged in the field of computational science and engineering, particularly in modeling, simulation, and optimization of nanoelectronics and very large scale integration (VLSI) circuits. In the fall of 2002, he was a Visiting Assistant Professor with the Department of Electrical and Computer Engineering, University of Massachusetts, Amherst. From 2003 to 2004, he was a Research Consultant with the System on a Chip ( $\mathrm{SoC})$ Technology Center, Industrial Technology Research Institute, Hsinchu. From 2003 to 2005, he was the Head of the Departments of Nanodevice and Computational Nanoelectronics, NDL, and during the fall of 2004, he became a Research Associate Professor with MISRC. From the fall of 2005 to the fall of 2008, he was an Associate Professor with the Department of Communication Engineering, NCTU, where he is currently a Full Professor with the Department of Electrical Engineering, is the Deputy Director of the Modeling and Simulation Center, and conducts the Parallel and Scientific Computing Laboratory. He is also the Deputy Director General of NDL. His current research areas include computational electronics and electromagnetics, the physics of semiconductor nanostructures, transport simulation and model parameter extraction for semiconductor and photonic devices, computer-aided-design theory and technology, biomedical and energy-harvesting device simulation, parallel and scientific computing, and optimization methodology. He has authored or coauthored over 150 research papers appearing in international book chapters, journals, and conferences. He has served as a Reviewer, Guest Associate Editor, Guest Editor, Associate Editor, and Editor for more than 30 international journals.

$\mathrm{Dr}$. $\mathrm{Li}$ is a member of Phi Tau Phi and is included in Who's Who in the World. He was the recipient of the 2002 Research Fellowship Award presented by the Pan Wen-Yuan Foundation, Taiwan, and the 2006 Outstanding Young Electrical Engineer Award from the Chinese Institute of Electrical Engineering, Taiwan. $\mathrm{He}$ has served as an active Reviewer for seven IEEE journals. He has also organized and served on several international conferences and was an Editor for the proceedings of international conferences.

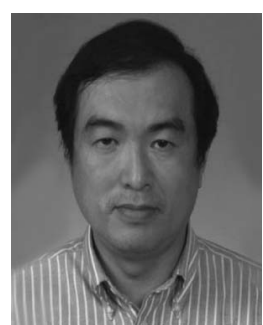

Edward Yi Chang (S'85-M'85-SM'04) received the B.S. degree from the Department of Materials Science and Engineering, National Tsing Hua University, Hsinchu, Taiwan, in 1977, and the Ph.D. degree from the Department of Materials Science and Engineering, University of Minnesota, Minneapolis, in 1985.

He was with Unisys Corporation GaAs Component Group, Eagan, MN, from 1985 to 1988 and with the Comsat Labs Microelectronic Group from 1988 to 1992 . He worked on GaAs MMIC programs on both groups. He was with National Chiao Tung University (NCTU), Hsinchu, in 1992. In 1994, he helped set up the first GaAs MMIC production line in Taiwan and became the President of Hexawave, Inc., Hsinchu, in 1995. He returned to the teaching position at NCTU in 1999 and is currently the Associate Dean of Engineering College. His research interests include new device and process technologies for compound semiconductor RFICs for wireless communication, III-V/Si integration for post CMOS application, and GaN Devices for RF and power applications.

Dr. Chang is a senior member and a Distinguished Lecturer of the IEEE Electron Devices Society. 